\title{
Telehealth for consultation and shoulder rehabilitation: a preliminary study on the perspectives of 30 patients during the COVID-19 lockdown
}

\author{
Dipit Sahu $^{1,2}$, Vaibhavi Rathod ${ }^{2}$, Ashish Phadnis ${ }^{1}$, Samarjit S. Bansal ${ }^{3}$ \\ ${ }^{1}$ Department of Orthopaedics, Jupiter Hospital, Thane, India \\ ${ }^{2}$ Mumbai Shoulder Institute, Mumbai, India \\ ${ }^{3}$ Department of Orthopaedics, Aastha Hospital, Mumbai, India
}

Background: This study aimed to determine: the perspectives of the patient cohort that underwent telehealth consultation and shoulder rehabilitation during coronavirus disease 2019 (COVID-19) and the differences in the patients' perspectives with the two different telehealth video applications (apps) used in the study.

Methods: This is a prospective study carried out during the COVID lockdown period of April to July 2020. Thirty consecutive patients from the orthopedics department of a tertiary institute in India underwent their first-ever session of a video app-based (Zoom or WhatsApp) telehealth consultation with shoulder rehabilitation exercises on a handheld mobile, tablet, or laptop device. After the virtual consultation, the patients were sent a validated telehealth usability questionnaire (TUQ) to evaluate their perspectives. Scores obtained from the TUQ were the primary outcome measure.

Results: The study was completed by 30 patients (16 men and 14 women) with an average age of 56 years (range, 20-77 years). The patients who contacted us during the lockdown period with either a stiff shoulder or a conservatively treated shoulder fracture were included in the study. The average TUQ score was 13.6 (median, 14.5; range, 6-21) out of a maximum of 21 points. Eighty percent of the patients were satisfied and found the telehealth service useful. Use of the Zoom app scored significantly higher (median, 17; average, 15.6) than the WhatsApp app (median, 8.5; average, 9.6) ( $\mathrm{p}=0.004)$.

Conclusions: Patients who received telehealth consultation and shoulder rehabilitation were overall satisfied. Telehealth apps with advanced video calling features such as Zoom should be preferred for higher patient satisfaction.

Keywords: Telerehabilitation; Rehabilitation; Telemedicine; COVID-19; SARS-CoV-2

\section{INTRODUCTION}

Around the months of February and March, 2020, several countries, including India, went into a total or partial lockdown and adopted strict social distancing measures to limit the spread of coronavirus disease 2019 (COVID-19) [1]. Any other non-urgent, non-traumatic problems that the patients were facing suddenly took a back seat due to the potential infectious and fatal nature of COVID-19 [2]. Accordingly, many countries' lockdown guidelines specified that patients should postpone any non-ur-

Received: April 19, $2021 \quad$ Revised: June 17, $2021 \quad$ Accepted: June 22, 2021

Correspondence to: Dipit Sahu

Department of Orthopaedics, Jupiter Hospital, Eastern Express Highway, Thane, Maharashtra 400601, India

Tel: +91-22-2172-5555, E-mail: E-mail: dip.it@me.com, ORCID: https://orcid.org/0000-0003-1888-4994

Financial support: None.

Conflict of interest: None. 
gent medical visits to free up hospital resources for tackling the COVID-19 pandemic [3]. The forced stay-at-home orders and workforce repurposing paved the way for an increase in the use of telehealth services for consultation, diagnostic, and rehabilitation services in orthopedics and fracture care. Some of our patients who had undergone shoulder surgery just before the lockdown was announced, and some newly diagnosed patients with shoulder problems, were unable to reach the hospital or any rehabilitation facility. Since telehealth was the newly preferred way of dispensing consultation and advisory services [4], we adopted that method to dispense consultation and shoulder rehabilitation, based on our pre-existing conventional protocols. The usage of telehealth services in the current pandemic continues to increase because it reduces unnecessary patient visits, maintains social distancing, and reduces the cost of health care.

However, despite this recently increasing trend, some surveys have raised doubts about the perceived benefits of telehealth because of problems such as audio-visual interruptions, unsatisfactory patient experience, and privacy concerns [5]. Recently published studies have also called for analyses of patient satisfaction with the telehealth model of delivering health care because there exist severe knowledge gaps regarding patients' perceptions and beliefs regarding telehealth services [6,7]. Therefore, we undertook this study with the aim to evaluate the perspectives of patients who used telehealth during COVID-19. We asked (1) what are the perspectives of the patients who underwent telehealth consultation and shoulder rehabilitation during COVID-19? and (2) are there any differences in the patients' perspectives with the two different telehealth applications (apps) used in the study? This second question was posed because we had initially recommended a universally available app but later switched to another one when the lockdown was partially relaxed.

\section{METHODS}

This study was approved by the Institutional Ethics Committee of Jupiter Hospital and all the patients gave informed consent to participate in the study and for the use of their photos.

\section{Study Setting and Design}

During India's lockdown period of April to July 2020, 30 consecutive patients were prospectively studied in the Shoulder division of the Orthopedics department in a tertiary care hospital of Mumbai. The patients underwent their first-ever video appbased telehealth consultation with shoulder rehabilitation exercises (as explained below) on a handheld mobile, tablet, or laptop device for 15-25 minutes, supervised by a physician (DS) and a physiotherapist (VR). The entire protocol of the virtual consultation and rehabilitation was similar to our in-person consultation sessions. After the virtual consultation, the patients were sent a validated telehealth usability questionnaire (TUQ) [8] to evaluate their perspectives on the usability of the telehealth services. The scores obtained from the TUQ formed our primary outcome measure. We used the strengthening the reporting of observational studies in epidemiology (STROBE) cohort reporting guidelines as a checklist [9].

\section{Participants/Study Subjects}

The patients who contacted us during the lockdown period with either a postoperative shoulder condition, a stiff shoulder (adhesive capsulitis as diagnosed by the senior shoulder surgeon), or a conservatively treated shoulder fracture were included in the study. The patients were required to have either a WhatsApp or Zoom app, on a mobile, tablet, or laptop device. All the patients had been seen by us face-to-us before the lockdown. For all the patients, the telehealth encounter was their first rehabilitation visit. The patients with acute shoulder pain, acute shoulder injury, or those who were approved for and awaiting a shoulder surgery were excluded.

\section{Telehealth Consultation}

The three-way (patient, DS, and VR) video telehealth consultation and rehabilitation was either on the WhatsApp app or the Zoom app, both of which allowed real-time video conferencing. The Zoom app requires a preinstallation learning tutorial on its installation and use; this was possible for our patients only during the latter time period (June and July) when the lockdown was partially relaxed and they could seek help from their relatives and family members. The WhatsApp video call can be activated by a simple call button and requires no learning tutorial. The Zoom video could be used on a mobile phone or a laptop device, but WhatsApp could be used only on a mobile phone.

The first 10 patients were consulted on WhatsApp call because of its nearly-universal accessibility and because no special training was needed for its use during the initial strict lockdown period of April and May. In June and July, we asked the 20 subsequent patients to seek help in downloading the Zoom app on either their laptop device (preferred) or on a tablet or mobile device. We had concerns triggered by the initial lower reliability scores of the first 10 patients who had used WhatsApp, and therefore we asked the next 20 patients to preferably use the Zoom app. Regulatory requirements under the Health Insurance Portability and Accountability Act (HIPAA) have been relaxed and the use of video calling apps such as WhatsApp, Zoom, 
Skype, and Facebook Messenger are allowed in compliance with the good faith provision of telehealth during COVID-19 [10]. Many other apps are also HIPAA-compliant, such as Skype for business, Cisco WebEx meeting, Amazon Chime, and GoToMeeting [11].

Prior to the telehealth call, the patients were sent a photo PDF file illustrating the steps of the exercises (Figs. 1-4). The patients were asked to sit on a chair at a distance of 6 feet from the mobile phone or laptop device; a phone had to be held by a family member who adjusted its position to center the patient image. The patients were taught the following exercises and were asked to repeat them 10 times. All four exercises were taught sequentially. Patient compliance to the exercise regimen was ensured by supervising them via the virtual platform once every month by the
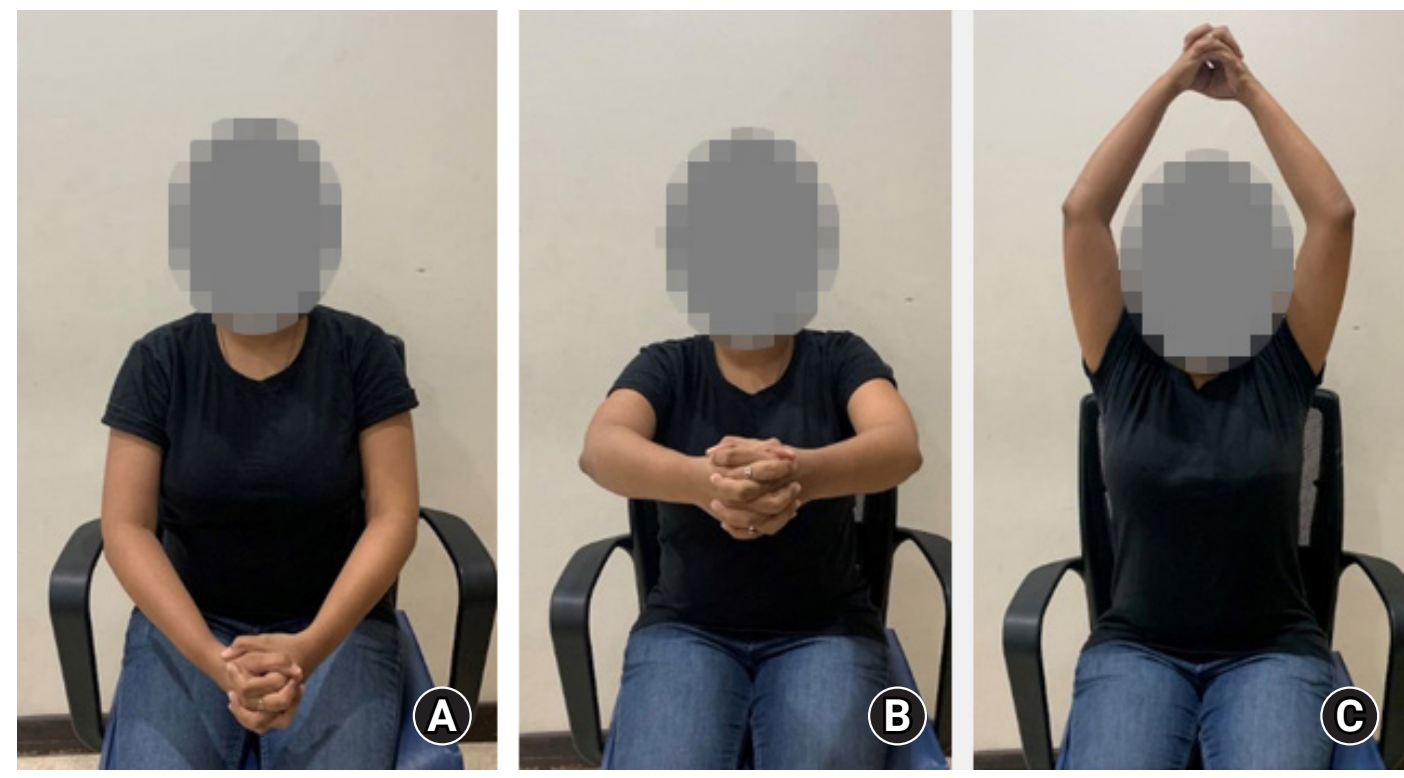

Fig. 1. Self-assisted elevation exercises with the patient sitting on a chair. (A) He/she interlocks their fingers and their elbows are flexed at $20^{\circ}-$ $30^{\circ}$, at rest the arms are internally rotated. (B) He/she slowly elevates both arms together in a controlled fashion. (C) He/she then fully elevates their arms together on top of the head, and in doing so the arms get externally rotated.
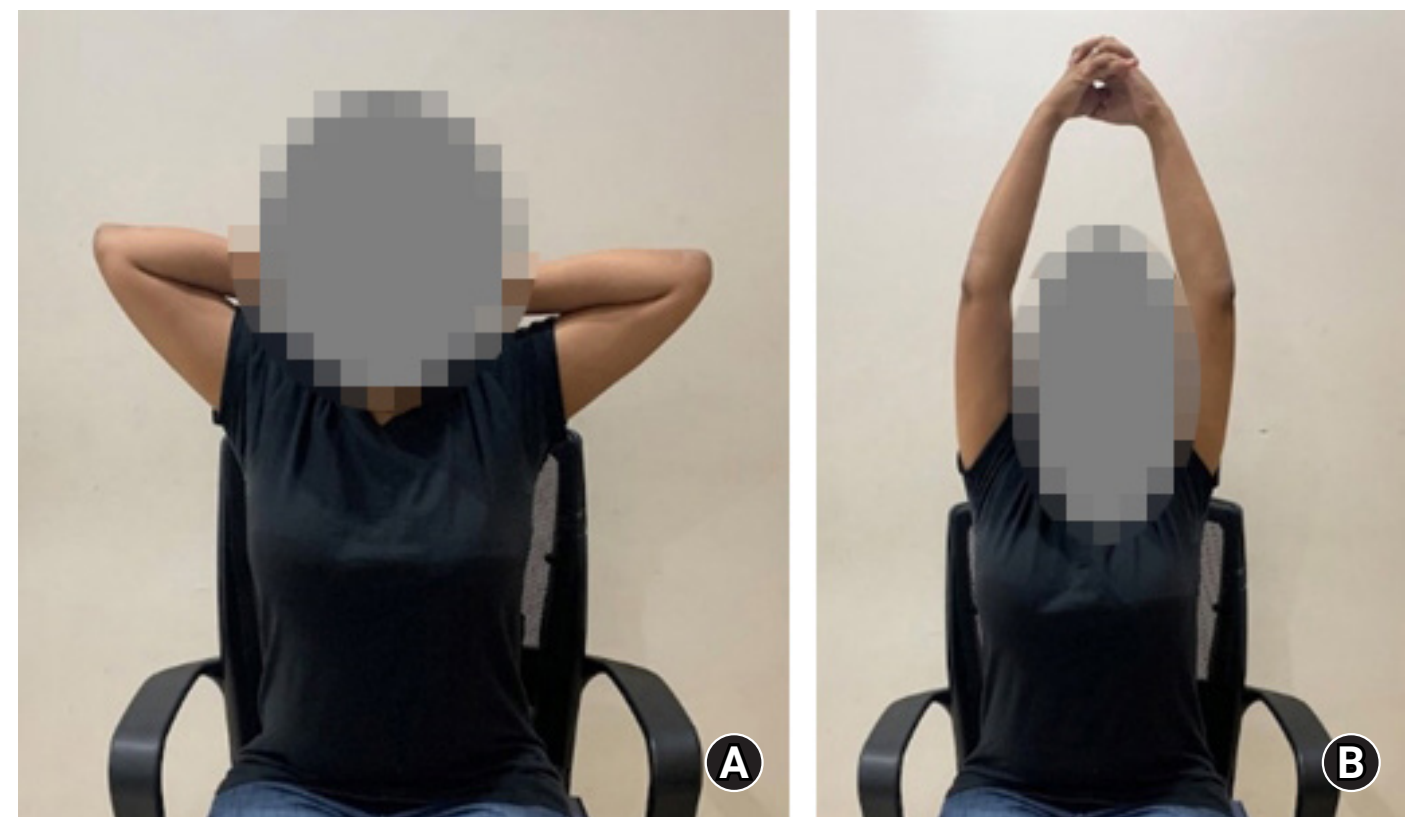

Fig. 2. External rotation with arm abducted (ER2) exercises with the patient sitting on a chair. (A) He/she puts their hands behind their head with the elbows externally rotated. (B) Then, he/she tries to elevate both arms above and behind their head. 

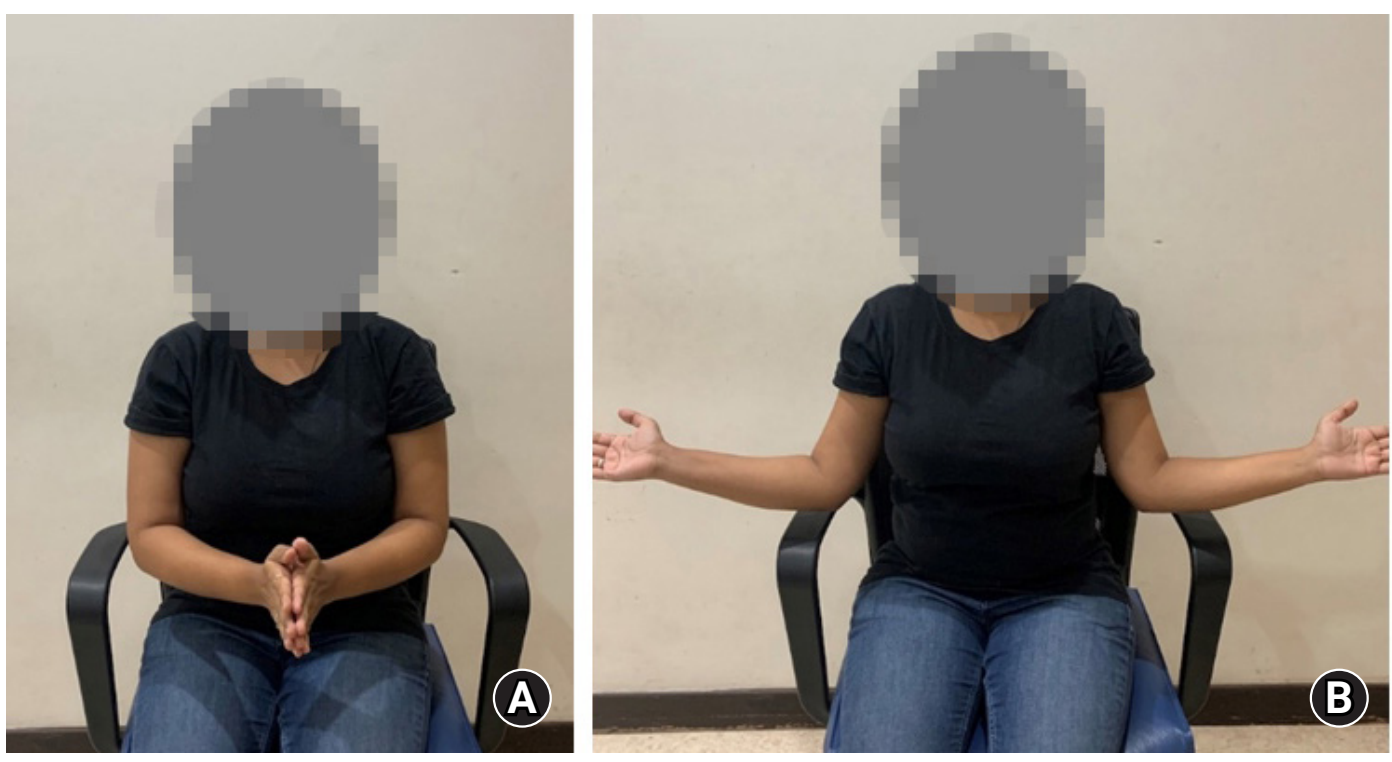

Fig. 3. External rotation of the arm with the elbow adducted (ER1) exercises with the patient sitting on a chair. (A) The patient joins their hands in the center. (B) Then, he/she takes their hands out by externally rotating their arms.
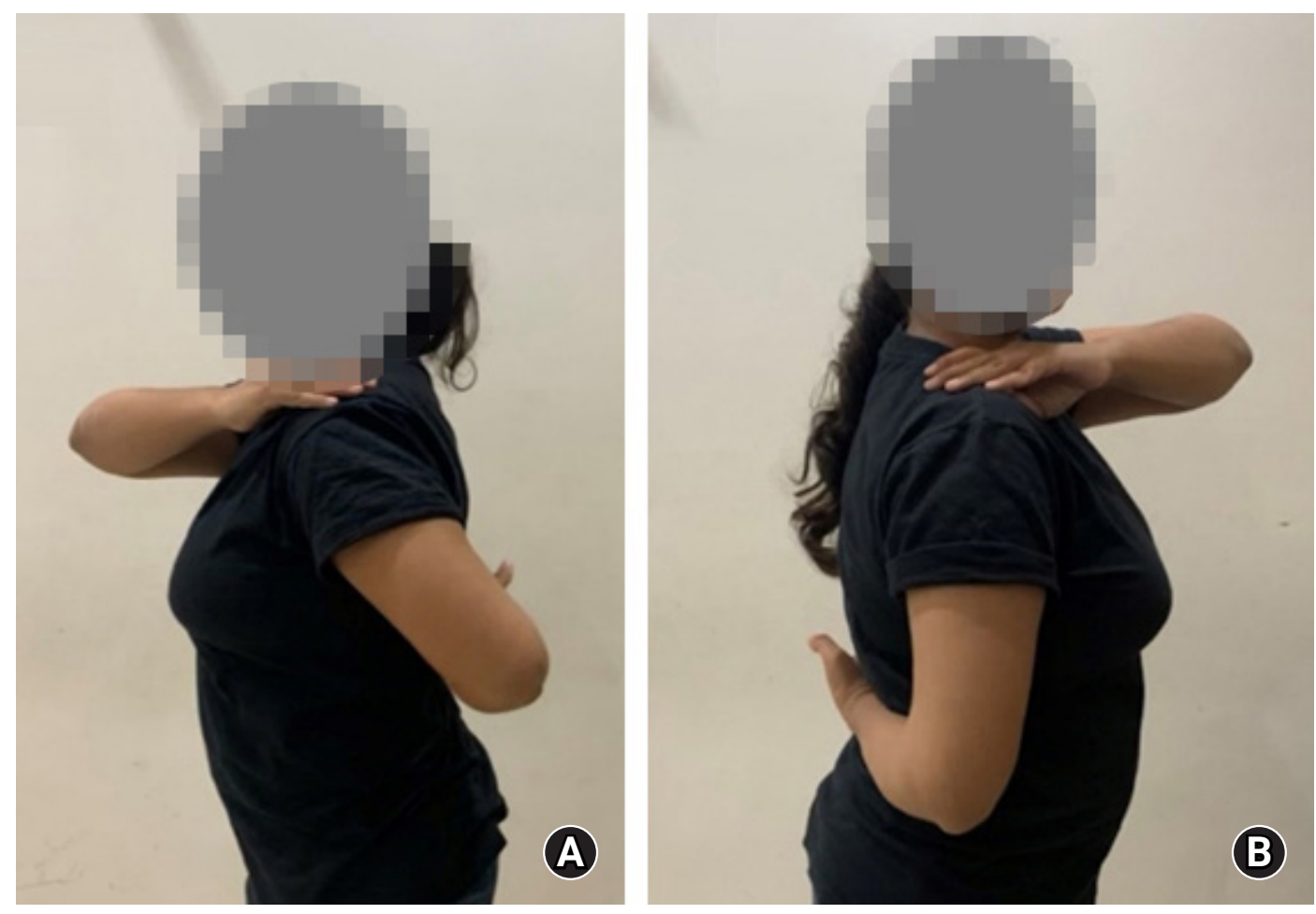

Fig. 4. Internal rotation exercises with the patient standing. (A) He/she places one hand over the opposite shoulder and the other hand behind their back. (B) Then, he/she changes the hands and performs the same action with the other hands on the opposite side.

physiotherapist and via face-to-face consultation with the physician once every 3 to 4 months or as was feasible in the lockdown situation.

\section{Rehabilitation Exercises}

As per our normal pre-existing protocol, all postoperative patients, patients with stiff shoulder due to capsulitis, and patients who were conserved for shoulder fractures (proximal humerus, greater tuberosity fracture, or clavicle fracture) underwent the 
same set of rehabilitation exercises. These exercises and their basic principles are widely published $[12,13]$ and have been in use for several years [13]. They are based on three fundamental principles: A. K. Saha's principle of zero position [14], Charles Neer principles of shoulder motion in scapular plane [15], and E. Amory Codman's principles of paradoxical motion [16,17] .

\section{Exercise protocol}

The above principles were applied in the following self-assisted rehabilitation exercises. (1) Self-assisted elevation exercises (Fig. 1): The patient is sitting on a chair with their back supported. He/ she interlocks their fingers and bends the elbows at an angle of $20^{\circ}-30^{\circ}$. In the resting position, the shoulder is internally rotated. Then the hands are moved overhead, in the scapular plane between the frontal and coronal plane. (2) External rotation with arm abducted (ER2) exercises (Fig. 2). First the hands are placed behind the head and then the elbows are externally rotated is externally rotated. Then, both hands are elevated together above the head, with the eventual aim to elevate them behind the head.

(3) External rotation of the arm with the elbow adducted (ER1)

(Fig. 3). (4) Internal rotation exercises (Fig. 4).

\section{Telehealth Usability Questionnaire}

The TUQ [8] is a comprehensive, validated questionnaire that can be used to evaluate a patient's perception of the usability of and satisfaction with various types of telehealth systems, including the traditional video systems as well as the newer generation of mobile devices. The questionnaire evaluates patients' perceptions in six domains (Table 1). (1) Usefulness, which is the patient's perception of how the system works overall to provide the health care service, and the advantages it offers in relation to traditional health care delivery; (2) ease of use and learnability, which refers to how easy it is to learn and use the system; (3) interface quality, which evaluates the interaction of the patient with the computer system and how easy it is to navigate the system with its graphical interface; (4) interaction quality, which evalu-

Table 1. Telehealth Usability Questionnaire items and the number of patients who chose those responses item within each domain

\begin{tabular}{|c|c|c|c|}
\hline \multirow{2}{*}{ Domain (item) } & \multicolumn{3}{|c|}{ No. of responses } \\
\hline & WhatsApp & Zoom & Combined \\
\hline \multicolumn{4}{|l|}{ Usefulness scale summary (1-3) } \\
\hline 1. Tele-rehabilitation improves my access to healthcare services. & 6 & 17 & 23 \\
\hline 2. Tele-rehabilitation saves me time traveling to a hospital or specialist clinic. & 8 & 18 & 26 \\
\hline 3. Tele-rehabilitation provides for my healthcare needs. & 3 & 14 & 17 \\
\hline \multicolumn{4}{|l|}{ Ease of use and learnability scale summary (4-6) } \\
\hline 4. It was simple to use this technology. & 5 & 20 & 25 \\
\hline 5. It was easy to learn to use the technology. & 7 & 16 & 23 \\
\hline 6. I believe I could become productive quickly using this technology. & 4 & 12 & 16 \\
\hline \multicolumn{4}{|l|}{ Interface quality scale summary $(7-10)$} \\
\hline 7. The way I interact with this application is pleasant. & 4 & 17 & 21 \\
\hline 8. I like using this application. & 2 & 13 & 15 \\
\hline 9. The application is simple and easy to understand. & 7 & 19 & 26 \\
\hline 10. This application is able to do everything I would want it to be able to do. & 3 & 9 & 12 \\
\hline \multicolumn{4}{|l|}{ Interaction quality scale summary (11-14) } \\
\hline 11. This application is able to do everything I would want it to be able to do. & 6 & 19 & 25 \\
\hline 12. I could hear the clinician clearly using this technology. & 6 & 16 & 22 \\
\hline 13. I felt I was able to express myself effectively. & 5 & 16 & 21 \\
\hline 14. Using the telehealth system, I could see the clinician as well as if we met in person. & 3 & 11 & 14 \\
\hline \multicolumn{4}{|l|}{ Reliability scale summary (15-17) } \\
\hline 15. I think the visits provided over the application are the same as in-person visits. & 3 & 13 & 16 \\
\hline 16. Whenever I made a mistake using the application, I could recover easily and quickly. & 5 & 11 & 16 \\
\hline 17. The application gave error messages that clearly told me how to fix problems. & 1 & 5 & 6 \\
\hline \multicolumn{4}{|l|}{ Satisfaction and future use scale summary $(18-21)$} \\
\hline 18. I feel comfortable communicating with the clinician using this technology. & 4 & 18 & 22 \\
\hline 19. Tele-rehabilitation is an acceptable way to receive healthcare services. & 5 & 16 & 21 \\
\hline 20. I would use tele-rehabilitation services again. & 3 & 15 & 18 \\
\hline 21. Overall, I am satisfied with tele-rehabilitation. & 6 & 18 & 24 \\
\hline
\end{tabular}


ates the interaction of the patient with the physician, including the audio-visual quality; (5) reliability, which refers to how easily the patient can recover from any error and whether the system offers steps on how to correct the error, and also whether the patient thinks the virtual visits are same as in-person visits; and (6) satisfaction and future use, which refer to the satisfaction with and the willingness of the patient to use the telehealth medium in the future. Each domain had three or four choices (Table 1), of which the patients could select all that applied to them. Each response was given a score of 1 and maximum possible choices was 21. A total score of $0-5$ was graded as poor, $6-10$ as fair, $11-15$ as good, and 16-21 as excellent.

\section{Patient Demographics}

The questionnaire was completed by 30 consecutive patients (16 men and 14 women), average age 56 years (range, 20-77 years) (Table 2). Ten patients were postoperative follow-ups (3-8 weeks postoperative), and 20 patients were in the conservatively treated group. One patient with adhesive capsulitis had been diagnosed with COVID-19 two weeks earlier, but had recovered and was still in home isolation. The rest of the 29 patients were never diagnosed with COVID-19.

\section{Variables and Outcome Measures}

The primary study variable was the TUQ score. The primary study outcome was to objectively evaluate the overall usability of the telehealth system, based on the average TUQ score and the individual response items chosen as a percentage of the maximum score. Our secondary study outcome was to evaluate the differences in the usability of Zoom and WhatsApp, based on the

Table 2. Demographics of the 30 patients in the study

\begin{tabular}{|c|c|c|c|c|}
\hline $\begin{array}{l}\text { Serial } \\
\text { number }\end{array}$ & Diagnosis/etiology & $\begin{array}{l}\text { Level of } \\
\text { education }\end{array}$ & $\begin{array}{l}\text { Device used for } \\
\text { telehealth }\end{array}$ & $\begin{array}{l}\text { Application used } \\
\text { for telehealth }\end{array}$ \\
\hline 1 & Post Latarjet surgery & Postgraduate & Mobile & WhatsApp \\
\hline 2 & Post Latarjet surgery & Graduate & Mobile & WhatsApp \\
\hline 3 & Post rotator cuff repair & Postgraduate & Mobile & WhatsApp \\
\hline 4 & Post Latarjet surgery & Graduate & Mobile & WhatsApp \\
\hline 5 & Post rotator cuff repair & High school & Mobile & WhatsApp \\
\hline 6 & $\begin{array}{l}\text { Postoperative follow-up of locked plate fixation of fracture dislocation of proximal } \\
\text { humerus }\end{array}$ & Graduate & Mobile & WhatsApp \\
\hline 7 & Post rotator cuff repair & High school & Mobile & WhatsApp \\
\hline 8 & Adhesive capsulitis & Postgraduate & Mobile & WhatsApp \\
\hline 9 & Adhesive capsulitis & Postgraduate & Mobile & WhatsApp \\
\hline 10 & Post rotator cuff repair & High school & Mobile & WhatsApp \\
\hline 11 & Postoperative follow-up locked plate fixation of fracture proximal humerus & Postgraduate & Laptop & Zoom \\
\hline 12 & Adhesive capsulitis & High school & Laptop & Zoom \\
\hline 13 & Adhesive capsulitis & Graduate & Laptop & Zoom \\
\hline 14 & Conserved fracture proximal humerus & High school & Laptop & Zoom \\
\hline 15 & Adhesive capsulitis & High school & Laptop & Zoom \\
\hline 16 & Adhesive capsulitis & High school & Laptop & Zoom \\
\hline 17 & Adhesive capsulitis & High school & Laptop & Zoom \\
\hline 18 & Conserved greater tuberosity fracture & Postgraduate & Laptop & Zoom \\
\hline 19 & Adhesive capsulitis & Postgraduate & Laptop & Zoom \\
\hline 20 & Adhesive capsulitis & Graduate & Mobile & Zoom \\
\hline 21 & Postoperative locked plate fixation of fracture proximal humerus & High school & Mobile & Zoom \\
\hline 22 & Adhesive capsulitis & Graduate & Mobile & Zoom \\
\hline 23 & Conserved fracture of midshaft clavicle & High school & Mobile & Zoom \\
\hline 24 & Adhesive capsulitis & Graduate & Mobile & Zoom \\
\hline 25 & Adhesive capsulitis & Postgraduate & Mobile & Zoom \\
\hline 26 & Adhesive capsulitis & High school & Mobile & Zoom \\
\hline 27 & Adhesive capsulitis & Graduate & Mobile & Zoom \\
\hline 28 & Adhesive capsulitis & Graduate & Mobile & Zoom \\
\hline 29 & Adhesive capsulitis & Postgraduate & Tablet & Zoom \\
\hline 30 & Adhesive capsulitis & Postgraduate & Tablet & Zoom \\
\hline
\end{tabular}


differences in their TUQ scores.

\section{Data Analysis}

IBM SPSS ver. 26 (IBM Corp., Armonk, NY, USA) was used for all statistical analyses. Descriptive statistics in the form of mean, median, standard deviation, and percentage of responses received were used to describe the TUQ. Secondarily, the average scores of the WhatsApp app users and the Zoom app users were also compared with the help of the Mann-Whitney U-test. A p-value of less than 0.05 was considered significant.

\section{RESULTS}

\section{Patients' Perspectives on Telehealth}

The average score of the 30 patients was 13.6 points (median, 14.5; range, 6-21 points) out of a maximum of 21 points (Table 3). This was graded as a good score. The domains of usefulness and ease of use/learning scored the maximum, with averages of $73.3 \%$ and $71 \%$, respectively. Interface quality, interaction quality, and satisfaction scored an average of $61.7 \%, 67.5 \%$, and $70.7 \%$, respectively, and reliability scored the least with $42.3 \%$ (Table 3 ).

Our evaluation of individual items in the TUQ revealed that most patients found the technology easy to learn to use (23/30), the app was easy and simple to understand (26/30), the process saved time and travel (26/30), they could hear the physician properly (22/30), and, overall, they were satisfied with the telerehabilitation (24/30). Fewer patients said that the visit over the app was similar to in-person visits (16/30), and error messages on how to correct a problem $(6 / 30)$ (Table 1$)$.

\section{Patients' Perspectives on the Two Different apps for Telehealth}

The Zoom app scored significantly higher (median, 17; average, 15.6) than the WhatsApp app (median, 8.5; average, 9.6) $(p=0.004)$. All the domains were scored higher by the Zoom users than WhatsApp users (Table 3).

\section{DISCUSSION}

Although telehealth usage has seen a major surge in the current COVID-19 pandemic, patient satisfaction remains a key component that will decide the future evolution of telehealth practices [18]. Therefore, the aim of this study was to evaluate patient perspectives regarding telehealth consultation and shoulder rehabilitation, and to evaluate the differences in perspectives with the use of two different apps. Our study found that the patients who underwent telehealth shoulder rehabilitation scored the use of telehealth services as good to excellent (Zoom) on the usability questionnaire. They also found the virtual interaction easy and useful. This will encourage more health care professionals to offer virtual care for shoulder rehabilitation and consultation through app-based calls, thus reducing the number of patient visits to health care facilities and cutting down their exposure during the pandemic and even in the foreseeable future.

In our study, the majority of patients found that the telehealth service saved them time and travel, and improved their access to health care services. Most patients also found that they were able to learn and use the technology easily, that they could understand the app easily, that they felt comfortable communicating with the clinician, and that they were overall satisfied with its use. It has been stated earlier in the literature that one of the advantages of this technology is that it saves time and travel for the patients [19]. The patients can avoid unnecessary travel to the health facility and can derive the benefits of consultation and rehabilitation sitting in their homes [19].

Our cohort included young and old patients, and patients with education levels ranging from high school to post-graduate. Hence, in our experience the benefits of telehealth can extend to

Table 3. Domains of the TUQ and the average score obtained in each domain by WhatsApp users, Zoom users, and combined

\begin{tabular}{lclcc}
\hline \multirow{2}{*}{ Subsets of TUQ } & Maximum score & \multicolumn{3}{c}{ Average score (\%) } \\
\cline { 3 - 5 } & & WhatsApp & $2.45(81.6)$ & $2.20(73.3)$ \\
\hline Usefulness & 3 & $1.70(56.6)$ & $2.40(80)$ & $2.13(71)$ \\
Ease of use and learnability & 3 & $1.60(53.3)$ & $2.90(72.5)$ & $2.47(61.7)$ \\
Interface quality & 4 & $1.60(40)$ & $3.05(76.2)$ & $2.70(67.5)$ \\
Interaction quality & 4 & $2.00(50)$ & $1.45(48.3)$ & $1.27(42.3)$ \\
Reliability & 3 & $0.90(30)$ & $3.35(83.7)$ & $2.83(70.7)$ \\
Satisfaction and future use & 4 & $1.80(45)$ & $15.60(74.2)$ & $13.60(64.7)$ \\
Total score & 21 & $9.60(45.7)$ & & \\
\hline
\end{tabular}

Values are presented as number (\%).

TUQ: telehealth usability questionnaire. 
patients of all ages and to patients with all levels of education. There have been doubts over the perceived benefits in the elderly age group [20], but in our experience this group of patients were the most satisfied and found the virtual interaction useful because it reduced their exposure and cut down on their unnecessary travel. Moreover, since our cohort included patients who had undergone surgery as well patients who were conservatively treated, this study shows that the benefits of telehealth can be extended to both categories of patients.

Audio-visual quality and internet connectivity are always a limiting factor in telehealth delivery [18], but in this study the interaction quality was found to be good by the majority of the patients. However, the reliability domain was scored the lowest (combined, 42\%; Zoom, 48\%; and WhatsApp, 30\%), which means that the apps did not give them correct error messages for fixing problems. Whenever the video was disconnected due to a weak network, the apps offered limited functions on the steps necessary for correction.

Overall, only $53 \%$ of the patients felt that the telehealth visits were the same as in-person visits. This is understandable because the physical reassuring touch of the physician is lacking in telehealth services, and some patients may still prefer to visit their health care provider in person [21]. Traditionally, telehealth has been used over larger platforms like Cisco-Tanberg, Polycom, Sony PCS 50, and Adobe Connect [8,22]. High patient satisfaction with telehealth has been demonstrated in earlier pre-COVID studies, but in those studies video conferences over traditional platforms were held at a regional medical center and with trained physicians [23]. Another recent study also evaluated patient satisfaction with phone calls and video calls [24], but it only assessed a limited number of parameters and it did not perform a comprehensive and detailed examination of the interactions, as was done in our study.

Our study demonstrated that the telehealth experience can be highly satisfying with the patients connecting from their homes over universally available mobile apps. Two other COVID-19 studies on patients with shoulder problems and their telehealth satisfaction have revealed similar results. Ben-Ari et al. [25] reported that the patients were highly satisfied with their telehealth encounters but had a significantly lower preference for future telehealth visits if there were no pandemic. Sabbagh et al. [26] reported that there was no difference in satisfaction between the telemedicine group and the in-person consultation group of patients who were evaluated as follow-ups after an earlier shoulder cuff repair or a shoulder arthroplasty. A study by Steele et al. [27] prior to the COVID pandemic also reported that patients were very satisfied with the telerehabilitation procedure.
The TUQ scores of the Zoom users were significantly higher than those of the WhatsApp users. The reason for this is because Zoom is an enterprise app with significantly advanced video calling features, while WhatsApp is a chat app with limited video calling features [28]. A higher percentage of Zoom users were satisfied (90\%) and were likely to use the service again (75\%), as compared to WhatsApp users ( $60 \%$ and $30 \%$, respectively).

This brings forth an important fact that the mobile apps chosen for telehealth will also influence patient perspectives. Telehealth is now being conducted over various apps, such as WhatsApp [29], which is a universally available chat app, and MyChart [7], which is a medical records app, but we recommend testing out any app in the first few calls to check the stability and smoothness of the audio-video quality over mobile as well as laptop devices.

Our study has several limitations. First, this study involved a small number of patients $(n=30)$ who evaluated a single virtual encounter. However, our sample size was comparable to that of other studies on telerehabilitation during non-COVID times $[22,30]$. The small sample size was intended to evaluate the patients' perspectives as a preliminary study before the scope of the services could be expanded further. Second, the outcomes of the rehabilitation and consultation via telehealth were not evaluated over the long term. Hence, any adverse effects of the telehealth visits could not be ascertained. However, it gave us significant insights in telehealth apps which may help us in further expanding the services and provide valuable lessons to physicians.

Third, this study included only those patients who underwent consultation and exercises for shoulder disorders. The results of the study can also be applied to upper limb and even lower limb disorders as the patient's interaction with the technology would remain the same. However, we are unable to comment on how patients who are bedridden, such as after spinal surgery, may perceive the telehealth services, because their interaction with the technology may be altered. Fourth, we did not compare the patients' perspectives between telehealth and conventional consultations. And finally, we did not randomize the use of the two telehealth apps between the users.

Patients who received home-based consultation and shoulder rehabilitation exercises via telehealth over video calling apps were satisfied overall and found that it saved them time and travel and improved their access to health care. We conclude that patients are also willing to use future telehealth services if they find them easy to learn and have good interaction quality, especially during this pandemic wherein the resources are constrained and patients' exposure to the hospitals needs to be reduced. A telehealth app with advanced video calling features and strong connectivity, 
such as the Zoom app, may provide better patient satisfaction as compared to an app with weaker video call connectivity such as WhatsApp.

\section{ORCID}

Dipit Sahu

Vaibhavi Rathod

\section{REFERENCES}

1. Azman AS, Luquero FJ. From China: hope and lessons for COVID-19 control. Lancet Infect Dis 2020;20:756-7.

2. Chang Liang Z, Wang W, Murphy D, Po Hui JH. Novel coronavirus and orthopaedic surgery: early experiences from Singapore. J Bone Joint Surg Am 2020;102:745-9.

3. British Orthopaedic Association. BOAST: management of patients with urgent orthopaedic conditions and trauma during the coronavirus pandemic [Internet]. London: British Orthopaedic Association; 2021 [cited 2021 Jul 30]. Available from: https://www.boa.ac.uk/resources/covid-19-boasts-combined1. html.

4. Tanaka MJ, Oh LS, Martin SD, Berkson EM. Telemedicine in the era of COVID-19: the virtual orthopaedic examination. J Bone Joint Surg Am 2020;102:e57.

5. Makhni MC, Riew GJ, Sumathipala MG. Telemedicine in orthopaedic surgery: challenges and opportunities. J Bone Joint Surg Am 2020;102:1109-15.

6. Murphy EP, Fenelon C, Murphy RP, et al. Are virtual fracture clinics during the COVID-19 pandemic a potential alternative for delivering fracture care? A systematic review. Clin Orthop Relat Res 2020;478:2610-21.

7. Siow MY, Walker JT, Britt E, et al. What was the change in telehealth usage and proportion of no-show visits for an orthopaedic trauma clinic during the COVID-19 pandemic. Clin Orthop Relat Res 2020;478:2257-63.

8. Parmanto B, Lewis AN Jr, Graham KM, Bertolet MH. Development of the telehealth usability questionnaire (TUQ). Int J Telerehabil 2016;8:3-10.

9. von Elm E, Altman DG, Egger M, et al. The strengthening the reporting of observational studies in epidemiology (STROBE) statement: guidelines for reporting observational studies. J Clin Epidemiol 2008;61:344-9.

10. Halawi MJ, Wang DD, Hunt TR 3rd. What's important: weathering the COVID-19 crisis: time for leadership, vigilance, and unity. J Bone Joint Surg Am 2020;102:759-60.

11. U.S. Department of Health \& Human Services. Notification of
Enforcement Discretion for Telehealth Remote Communications During the COVID-19 Nationwide Public Health Emergency [Internet]. Washington, DC: U.S. Department of Health \& Human Services; 2020 [cited 2021 Jul 30]. Available from: https://www.hhs.gov/hipaa/for-professionals/special-topics/ emergency-preparedness/notification-enforcement-discretion-telehealth/index.html.

12. Roulet S, Borel F, Franger G, Liotard JP, Michelet A, Godenèche A. Immediate self-rehabilitation after open Latarjet procedures enables recovery of preoperative shoulder mobility at 3 months. Knee Surg Sports Traumatol Arthrosc 2019;27:3979-88.

13. Liotard J, Walch G. 1985-2015: Comment et pourquoi 1' automobilisation précoce après une réparation de coiffe ? In: Vuillemin V, Lapègue F, Collin P, editors. L'épaule du classique à linédit. Montpellier: Sauramps Medical; 2015. p. 1-16.

14. Ozaki J, Kawamura I. “Zero-position” functional shoulder orthosis. Prosthet Orthot Int 1984;8:139-42.

15. Neer CSI. Shoulder reconstruction. 1st ed. Philadelphia, PA: WB Saunders Company; 1990. p. 2-7.

16. Codman EA. The shoulder; rupture of supraspinatus tendon and other lesions in or about the subacromial bursa. Boston, MA: T. Todd Company; 1934. p. 513.

17. Mallon WJ. On the hypotheses that determine the definitions of glenohumeral joint motion: with resolution of Codman's pivotal paradox. J Shoulder Elbow Surg 2012;21:e4-19.

18. Lanham NS, Bockelman KJ, McCriskin BJ. Telemedicine and orthopaedic surgery: the COVID-19 pandemic and our new normal. JBJS Rev 2020;8:e2000083.

19. Azma K, RezaSoltani Z, Rezaeimoghaddam F, Dadarkhah A, Mohsenolhosseini S. Efficacy of tele-rehabilitation compared with office-based physical therapy in patients with knee osteoarthritis: a randomized clinical trial. J Telemed Telecare 2018; 24:560-5.

20. Seto E, Smith D, Jacques M, Morita PP. Opportunities and challenges of telehealth in remote communities: case study of the Yukon Telehealth System. JMIR Med Inform 2019;7:e11353.

21. Chwistek M. "Are you wearing your white coat?": telemedicine in the time of pandemic. JAMA 2020;324:149-50.

22. Eriksson L, Lindström B, Ekenberg L. Patients' experiences of telerehabilitation at home after shoulder joint replacement. J Telemed Telecare 2011;17:25-30.

23. Sinha N, Cornell M, Wheatley B, Munley N, Seeley M. Looking through a different lens: patient satisfaction with telemedicine in delivering pediatric fracture care. J Am Acad Orthop Surg Glob Res Rev 2019;3:e100.

24. Rizzi AM, Polachek WS, Dulas M, Strelzow JA, Hynes KK. The new 'normal': rapid adoption of telemedicine in orthopaedics 
during the COVID-19 pandemic. Injury 2020;51:2816-21.

25. Ben-Ari E, Kirschenbaum J, Patel RG, et al. The future of health care service in orthopedic practice: telemedicine or in-person visits? J Shoulder Elbow Surg 2021 Jun 2 [Epub]. https://doi. org/10.1016/j.jse.2021.05.008.

26. Sabbagh R, Shah N, Jenkins S, et al. The COVID-19 pandemic and follow-up for shoulder surgery: the impact of a shift toward telemedicine on validated patient-reported outcomes. J Telemed Telecare 2021 Feb 1 [Epub]. https://doi.org/10.1177/1357633 X21990997.

27. Steele L, Lade H, McKenzie S, Russell TG. Assessment and diagnosis of musculoskeletal shoulder disorders over the Internet. Int J Telemed Appl 2012;2012:945745.
28. Sawers P. WhatsApp is addressing group video-calling limitations to better compete with Zoom [Internet]. San Francisco, CA: VentureBeat; 2020 [cited 2021 Jul 30]. Available from: https://venturebeat.com/2020/04/21/whatsapp-is-addressinggroup-video-calling-limitations-to-better-compete-withzoom/.

29. Pandey N, Srivastava RM, Kumar G, Katiyar V, Agrawal S. Teleconsultation at a tertiary care government medical university during COVID-19 Lockdown in India: a pilot study. Indian J Ophthalmol 2020;68:1381-4.

30. Tousignant M, Giguère AM, Morin M, Pelletier J, Sheehy A, Cabana F. In-home telerehabilitation for proximal humerus fractures: a pilot study. Int J Telerehabil 2015;6:31-7. 